\title{
Supersonic Compressor Cascade Shape Optimization under Multiple Inlet Mach Operating Conditions
}

\author{
Marco Casoni * ${ }^{*}$, Andrea Magrini $(\mathbb{B}$ and Ernesto Benini $\mathbb{1}$ \\ Department of Industrial Engineering, University of Padova, via Venezia 1, 35131 Padova, Italy; \\ andrea.magrini@phd.unipd.it (A.M.); ernesto.benini@unipd.it (E.B.) \\ * Correspondence: marco.casoni@studenti.unipd.it
}

Received: 9 May 2019; Accepted: 28 May 2019; Published: 30 May 2019

check for updates

\begin{abstract}
Transonic compressors are widely used today in propulsion and industrial applications thanks to their higher specific work compared to subsonic. In this work, the aerodynamic optimization of a two-dimensional Computational Fluid Dynamics (CFD) model of the transonic cascade ARL-SL19 is described. The validated computational model is used for a multi-objective optimization of the cascade at three different inlet Mach numbers using a genetic algorithm and an artificial neural network, with the aim of reducing total pressure loss and increasing maximum pressure ratio. Finally, the optimized shapes on the Pareto fronts are investigated, analyzing mechanisms responsible for loss reduction and enhanced compression. Profiles having the lowest losses have flatter camberlines and reduced acceleration of flow on the suction side, while geometries achieving the highest pressure ratio values have a more cambered shape with a concave suction side.
\end{abstract}

Keywords: transonic compressor; ARL-SL19 cascade; turbomachinery optimization; genetic algorithm; Computational Fluid Dynamics (CFD)

\section{Introduction}

Modern compressors and fans have tip sections of the blades typically operating at supersonic relative inlet velocity and subsonic outlet velocity; ultimately, this leads to fewer compressor stages and lighter, more compact propulsors. At supersonic inlet conditions, the flow field is characterized by a complex shock wave system, whose strength and interaction with the boundary layer largely affects pressure rise and entropy generation. Moreover, these phenomena strongly deteriorate the performance at off-design conditions and reduce surge and rotating stall margins [1]. For these reasons, the design of supersonic blades is critical, and new shapes are designed ad hoc for given operative conditions, rather than chosen from a family of profiles. Nowadays, three-dimensional aerodynamic models, in which the entire blade is simulated, are widely used; nevertheless, two-dimensional analysis is still fundamental in blade design and can provide extremely accurate results, more difficultly matched by more complex simulations. Computational Fluid Dynamics (CFD) in conjunction with automatic methods has been widely used in numerical simulations and optimization of supersonic blades. In [2], a multi-objective optimization of a 2D S-shaped supersonic profile, operating at a unique incidence condition, was conducted using a Kriging-assisted evolutionary algorithm in order to minimize total pressure losses and to maximize the pressure ratio. In [3,4], one-dimensional analytical shock-loss models were developed to predict overall total pressure losses. Upon these models, optimizations were conducted to reduce losses on two baseline geometries, acting on profile shape and thickness distribution. In [5], an optimization based on differential evolution was carried out to reduce losses in a supersonic blade, parametrizing the camberline only. A numerical investigation on potential improvement of the transonic compressor by using tandem rotor blades was carried out in [6]. In [7], a 3D optimization of tandem rotors, National Air and Space Administration (NASA) Stage 35 was carried out using 
the Non-dominated Sorting Genetic Algorithm (NSGA II) and the backpropagation Artificial Neural Network (ANN) technique to maximize the total pressure ratio and reduce the total pressure loss coefficient. Typical improvements achieved in various optimizations were around $20 \%$ for efficiency and 3\% regarding the pressure ratio. This paper presents a multi-objective multi-point aerodynamic optimization of a two-dimensional supersonic cascade that minimizes the total pressure-loss coefficient and maximizes the pressure ratio. The computational two-dimensional model was validated upon experimental data collected on a linear cascade in supersonic wind tunnel tests. The optimization framework comprised a genetic multi-objective algorithm and an iterative automatic procedure to calculate fitness functions. An artificial neural network was used to conduct a meta-model-based optimization to try and enlarge the Pareto fronts. Optimized profiles were investigated by the means of isentropic Mach number, Mach contours, and total pressure contours.

\section{Baseline Cascade Modeling and Validation}

The ARL-SL19 cascade, investigated by Schreiber and Starken in [8] and by Liu in [3], is a state-of-the-art geometry for supersonic cascade design. In the present study, it was taken as the baseline to implement shape modification so as to enhance cascade performances. The selected cascade belongs to the category of low-turning supersonic reaction cascades: it makes use of an S-shaped profile, implementing the compression via oblique shock waves attached to the leading edge, and an external pre-compression mechanism induced by the concave front portion of the suction side. In addition, it is of particular interest since it was especially designed for investigation of strong Shock Wave-Boundary Layer Interaction (SWBLI). This cascade typically operates at Unique Incidence (UI), a condition that relates the inlet Mach number to the inlet flow angle, widely described in [9-11]. Such a design is typical of tip sections of highly-loaded transonic fans with a nominal relative inlet Mach number of 1.61 and an axial Mach number of 0.87, providing a static pressure ratio of more than 2.4 with little flow turning. Experimental data, upon which the validation of the numerical model was carried out, were collected in a supersonic wind tunnel test at the Deutsche Zentrum für Luft und Raumfahrt (DLR) [8]. The object of the test was a statoric cascade consisting of five ARL-SL19 profiles with the geometrical features reported in Table 1.

Table 1. Geometric features of the experimental setup for the ARL-SL19 cascade.

\begin{tabular}{ccc}
\hline Parameter & Symbol & Value \\
\hline Number of blades & $n$ & 5 \\
Chord & $c(\mathrm{~mm})$ & 85 \\
Pitch & $s(\mathrm{~mm})$ & 55.58 \\
Solidity & $\sigma$ & 1.5294 \\
Stagger angle & $\lambda(\mathrm{deg})$ & 56.93 \\
\hline
\end{tabular}

Some tests were conducted with different Axial Velocity-Density Ratios (AVDR), assuring that the cascade operated in unique incidence conditions. The Reynolds number was kept $1.12 \times 10^{6}$ in all the experiments; total temperature and pressure were maintained constant upstream of the test section, as well, with values ranging in the intervals $T_{0}=300 \div 312 \mathrm{~K}$ and $p_{0}=1 \div 1.3 \mathrm{~atm}$. Typical flow quantities are summarized in Table 2. 
Table 2. Flow quantities in the experimental tests.

\begin{tabular}{cccccc}
\hline Parameters & Symbol & A & B & C & D \\
\hline Inlet Mach number & $M_{1}$ & 1.58 & 1.58 & 1.59 & 1.59 \\
Inlet flow angle & $\beta_{1}(\mathrm{deg})$ & 57.9 & 57.9 & 57.9 & 57.9 \\
Outlet Mach number & $M_{2}$ & 0.93 & 0.91 & 0.93 & 0.91 \\
Outlet flow angle & $\beta_{2}(\mathrm{deg})$ & 61.2 & 60.8 & 58 & 57.5 \\
AVDR & $A V D R$ & 0.99 & 1 & 1.12 & 1.14 \\
Loss coefficient & $\Omega$ & 0.144 & 0.143 & 0.123 & 0.117 \\
Pressure ratio & $\pi_{c}$ & 2.12 & 2.16 & 2.18 & 2.22 \\
\hline
\end{tabular}

The test B in Table 2 with AVDR = 1 was chosen as the baseline case to be replicated in the validation. In such a condition, the flow was stationary, and since all the relevant quantities were measured on the simply-extruded central blade of the row, three-dimensional effects and sidewall influence were minimized. The profile, generated using a Catmull-Rom curve and semi-circular arcs for leading and trailing edges, has been inserted into a two-dimensional structured multi-block domain, extending for $1 \times c_{a x}$ upstream of the leading edge and $4 \times c_{a x}$ downstream of the trailing edge, where $c_{a x}$ is the axial projection of the chord. Around the profile, an O-grid was extruded with the number of inflation layers ranging from 100-150 depending on the grid refinement and an exponential growth rate from 1.04-1.08. The first layer height was fixed in order to get an $Y^{+}<1$, to ensure an adequate resolution in the calculation of velocity profiles inside the boundary layer using wall functions. Three different grid sizes were tested, ranging from 100k-600k cells. In Tables 3 and 4, and Figures 1 and 2, the main features of the three grids and quality parameters are reported.

Table 3. Features of the mesh.

\begin{tabular}{ccccc}
\hline Entity & Symbol & Coarse & Medium & Fine \\
\hline Inlet/outlet & N1 & 113 & 165 & 328 \\
Periodic inlet & N2 & 50 & 75 & 100 \\
Periodic middle & N3 & 205 & 267 & 537 \\
Periodic outlet & N4 & 150 & 230 & 260 \\
Inner O-grid tang & N5 & 568 & 758 & 1347 \\
Inner O-grid rad & N6 & 100 & 100 & 150 \\
Inner O-grid Layer 1 (mm) & Y1 & $8.5 \times 10^{-5}$ & $8.5 \times 10^{-5}$ & $8.5 \times 10^{-5}$ \\
Outer O-grid rad & N7 & 50 & 60 & 100 \\
Y+ & Y+ & 0.05 & 0.05 & 0.05 \\
Growth rate O-grid & G & 1.08 & 1.08 & 1.04 \\
Cell number & $\mathrm{C}$ & 115,809 & 189,005 & 596,605 \\
\hline
\end{tabular}

Table 4. Quality parameters of the mesh.

\begin{tabular}{cccc}
\hline Quality Parameters & Coarse & Medium & Fine \\
\hline Max skewness equiangle & 0.45798 & 0.4136 & 0.4152 \\
Max area ratio & 3.001 & 4.3937 & 2.7704 \\
Min included angle (deg) & 48.9 & 52.7 & 52.7 \\
\hline
\end{tabular}




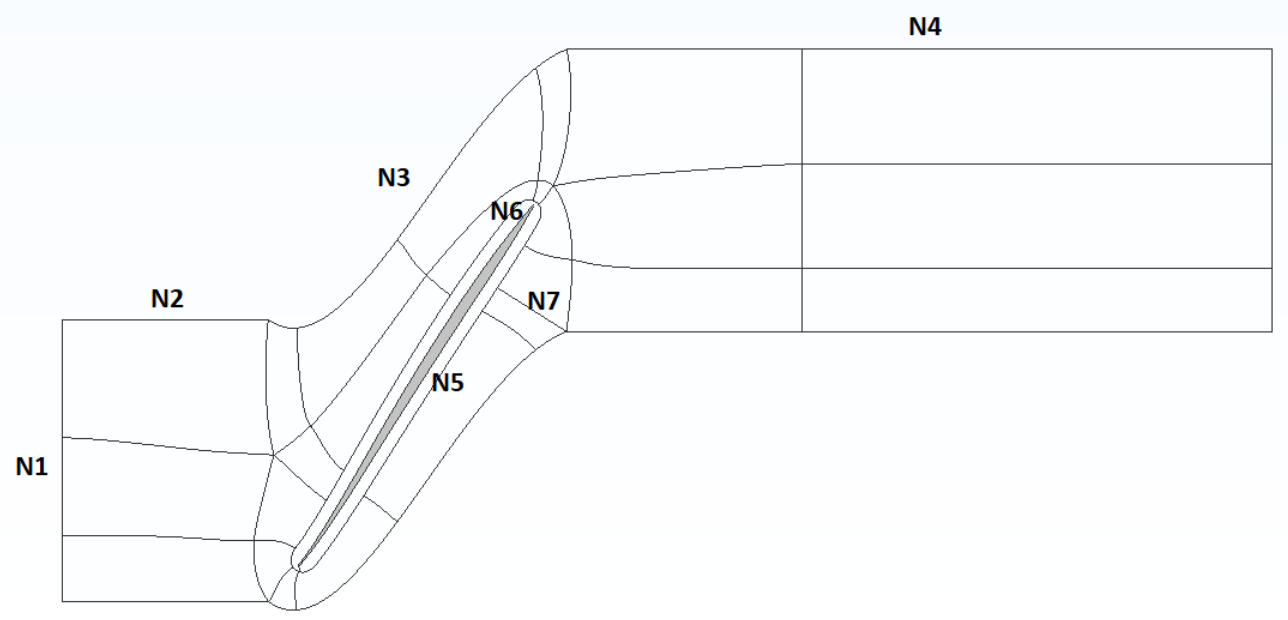

Figure 1. Scheme of the multi-block grid.
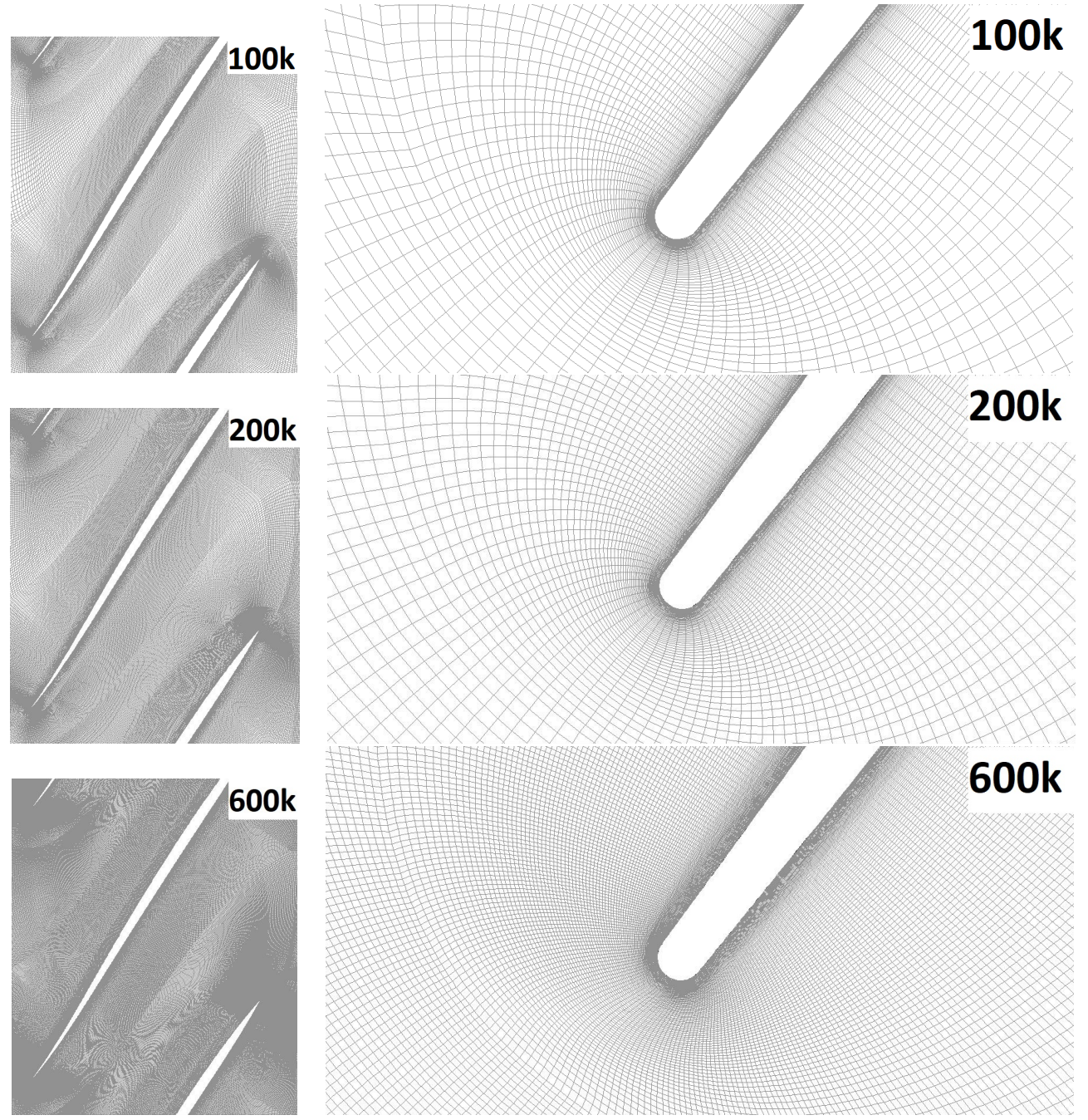

Figure 2. Blade passage and detail of the leading edge of the three grid refinements.

Calculations were carried out using ANSYS ${ }^{\mathrm{TM}}$ Fluent v16 [12], in which the Reynolds Averaged Navier-Stokes (RANS) equations coupled with a turbulence model were solved using a finite volume method approach. The solver is pressure based with a coupled scheme of resolution and Green-Gauss cell-based spatial discretization. As for the boundary conditions, a pressure far field at the inlet, 
with specifications of the turbulence intensity and viscosity ratio, was prescribed. Total temperature and pressure at the inlet were fixed respectively at $T_{0}=310 \mathrm{~K}$ and $p_{0}=1 \mathrm{~atm}$; in this way, the resulting Reynolds number was $R e=1.17 \times 10^{6}$, close to the one obtained in the experiments. At the outlet, the pressure outlet was imposed; in particular, $p_{2}=2.16 \times p_{1}$ was set during the validation phase. Blade walls were treated as walls with no slip; for the upper and lower bounds of the domain, periodic translational was prescribed in order to simulate an infinite blade row. Convergence was established when all residuals went under $10^{-5}$, and the oscillation of the inlet Mach number, inlet flow angle, and loss coefficient were below a certain threshold. Five turbulence models were tested: $k-\omega$ Shear Stress Transport (SST) [13], $k-\epsilon$ standard (STD) [14], $k-\epsilon$ realizable (REAL) [15], $k-\epsilon$ Re-Normalisation Group (RNG) [16], and Spalart-Allmaras (SA) [17] with the 100k grid. For every simulation, conditions at infinity were tuned in such a way that they could respect the experimental Mach number in the inlet section. In fact, an arbitrary choice of the inlet flow angle at infinity will generally modify the couple $\left(M_{1}, \beta_{1}\right)$ at the inlet, according to the unique incidence. In Table 5, global quantities are reported as the mass-weighted average in the section of interest for each simulated model of turbulence, while Figure 3 shows the isentropic Mach distribution.

Table 5. Global quantities in the experimental test and calculated with different turbulence models.

\begin{tabular}{ccccccc}
\hline Model & Experimental Data & $k-\omega$ SST & $k-\epsilon$ STD & $k-\epsilon$ REAL & $k-\epsilon$ RNG & SA \\
\hline$M_{1}$ & 1.58 & 1.58 & 1.58 & 1.58 & 1.58 & 1.58 \\
$\beta_{1}(\mathrm{deg})$ & 57.9 & 58.40 & 58.53 & 58.69 & 58.50 & 58.38 \\
$M_{2}$ & 0.91 & 0.912 & 0.897 & 0.900 & 0.902 & 0.906 \\
$\beta_{2}(\mathrm{deg})$ & 60.8 & 61.56 & 61.07 & 61.31 & 61.22 & 61.31 \\
$A V D R$ & 1 & 1 & 1 & 1 & 1 & 1 \\
$\Omega$ & 0.143 & 0.1342 & 0.1550 & 0.1511 & 0.1484 & 0.1406 \\
$\pi_{c}$ & 2.16 & 2.16 & 2.16 & 2.16 & 2.16 & 2.16 \\
\hline
\end{tabular}

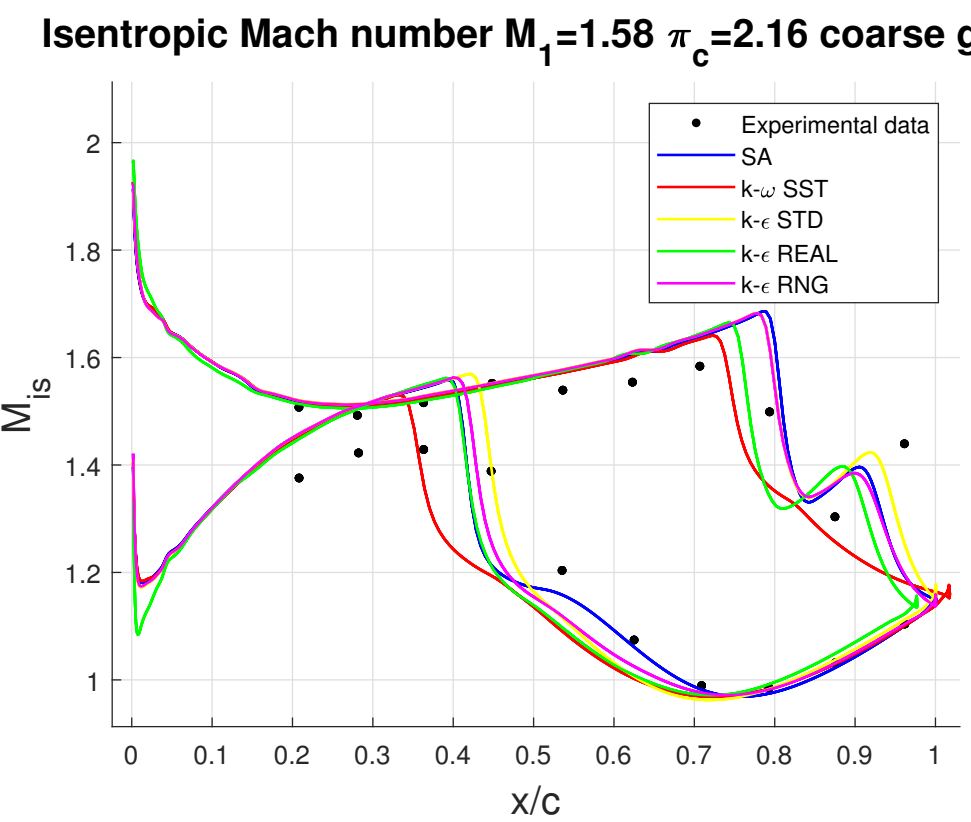

Figure 3. Isentropic Mach number: experimental data and numerical calculations with different turbulence models.

It can be noticed that the inlet flow angle calculated in the simulations was overestimated by about $0.5^{\circ}$, and the same applies for the exit flow angle, probably because of the lack of accurate knowledge of the leading edge geometry. However, the mean flow deflection was well captured by the SA model and slightly less by $k-\omega$ SST and $k-\epsilon$ RNG. The loss coefficient was overestimated for $k-\epsilon$ variants, while it was underestimated for the $k-\omega$ SST. The SA model was able to get closer to the experimental 
$\Omega$, with a slight underestimation. $k-\epsilon$ and the SA model replicated with better accuracy the isentropic Mach number distribution, with little anticipation of the point of incidence of the reflected shock on the suction side, while the $k-\omega$ SST model was not able to catch the re-acceleration of the flow near the trailing edge. $k-\epsilon$ RNG and the SA model seemed to provide better consistency with the experimental calculated loss coefficient, the isentropic Mach number distribution, and flow angles, with a slightly better agreement for the SA. In a previous work related to the same cascade [18], the authors found that the SA model could provide an overall good match with experimental data, with similar results for $k-\epsilon$. However, for the current case, the $k-\epsilon$ RNG model was adopted for its closer prediction of $\Omega$ at validation conditions and its high responsiveness to the effects of rapid strain and streamline curvature [12], favorable attributes for the simulation of hooked configurations possibly arising during optimization. A sensitivity analysis towards the grid size was also carried out: two levels of grid refinement were tested, namely a 200k cell mesh and a 600k one. Results are summarized in Table 6 and Figure 4. It can be noted that global quantities remained substantially unchanged with respect to grid refinement, with a slightly better agreement in the loss coefficient. However, the decrease in the acceleration on the suction side near the trailing edge departed from experimental data. This small sensitivity of numerical quantities towards grid refinement suggested that the coarse grid had sufficient accuracy to ensure reliable results, with the remarkable advantage of a faster calculation over finer grids. For these reasons, it was adopted for the optimization, given the large number of simulations requested for each evaluation of the fitness functions.

Table 6. Global quantities with different grid refinements.

\begin{tabular}{ccccc}
\hline Quantities & Experimental Data & Coarse & Medium & Fine \\
\hline$M_{1}$ & 1.58 & 1.58 & 1.58 & 1.58 \\
$\beta_{1}(\mathrm{deg})$ & 57.9 & 58.50 & 58.50 & 58.50 \\
$M_{2}$ & 0.91 & 0.902 & 0.902 & 0.902 \\
$\beta_{2}(\mathrm{deg})$ & 60.8 & 61.31 & 61.24 & 61.22 \\
$\Omega$ & 0.144 & 0.1484 & 0.1479 & 0.1472 \\
\hline
\end{tabular}

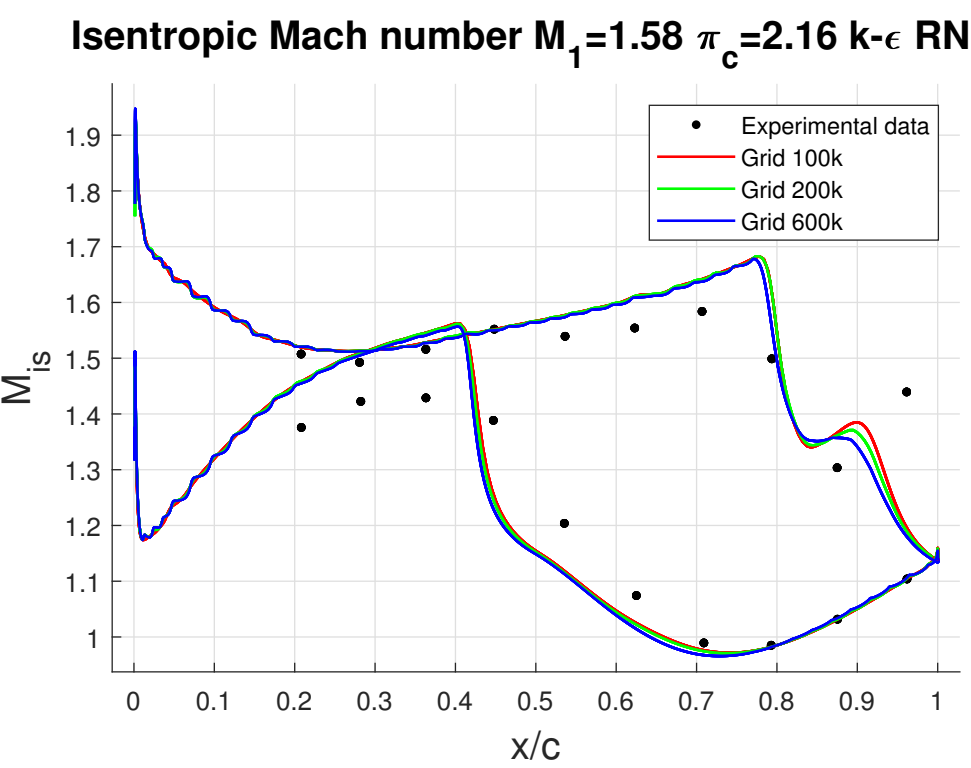

Figure 4. Isentropic Mach number: experimental data and numerical calculations with different grid refinements. $k-\epsilon$ RNG model.

\section{Optimization Procedure}

The aim of the multi-objective optimization problem was to minimize the two objective functions: 


$$
\begin{aligned}
& f_{1}=\Omega \text { at } p_{2 \max } \\
& f_{2}=\frac{1}{p_{2 \max }}
\end{aligned}
$$

where $p_{2 \max }$ is the maximum reachable back-pressure before the Spill Point (SP), and therefore proportional to the maximum effective pressure ratio $\pi_{c}$. The former objective is the loss coefficient in UI calculated right before the unstarted condition. It is indubitably of major concern in a supersonic cascade, given its ingrained ability to generate high pressure ratios thanks to the high kinetic load of the flow. Nevertheless, the second objective maintains a certain relevance: First of all, by converting part of the work exchanged by a rotor blade in pressure energy, losses due to high velocity diffusion in the stator blade are reduced. Furthermore, a higher maximum back-pressure achievable in unique incidence establishes a wider margin for SP. The spilled condition, described in [10], leads to detached passage shocks, to a decrease in the effective pressure ratio, and to an increased loss coefficient. In [1], numerical simulations investigated stall phenomena in rotor blades and showed how the spilled condition can interfere with the tip clearance vortex, leading to the rotating stall insurgency.

\subsection{Parameterization of the Profile}

The parameterization was carried out using a Bézier curve, in view of its regularity. Only the first half of the camberline was parameterized, while the thickness was kept unchanged, in order not to alter the structural properties of the airfoil significantly. This choice was due to the limited influence of the trailing fraction of the profile on performance, because in typical operating conditions, pressure rise is strongly related to the wave pattern in the fore part of the profile, while the rear fraction is subjected to highly turbulent flow. The work in [2], for example, showed that the difference between full and half camberline parameterization was around $5 \%$ in the loss coefficient. In the present work, six control points were employed for the Bézier curve, with the first four having fixed a X-coordinate and a variable $\mathrm{Y}$-coordinate and the fifth having a variable $\mathrm{X}$-coordinate and the ordinate computed according to:

$$
y_{5}=y_{6}+\left(x_{5}-x_{6}\right) \times t
$$

where $t$ is the tangent of the rear half of the camberline in the contact point with the front half, ensuring $C(1)$ continuity between the two portions. The thickness distribution, computed normal to the camberline, was kept fixed. Figure 5 shows the parameterized profile and Bézier polygon. No constraints were used, except for bounds on the control point displacement to provide the search algorithm with the highest exploration freedom without generating excessively warped shapes.

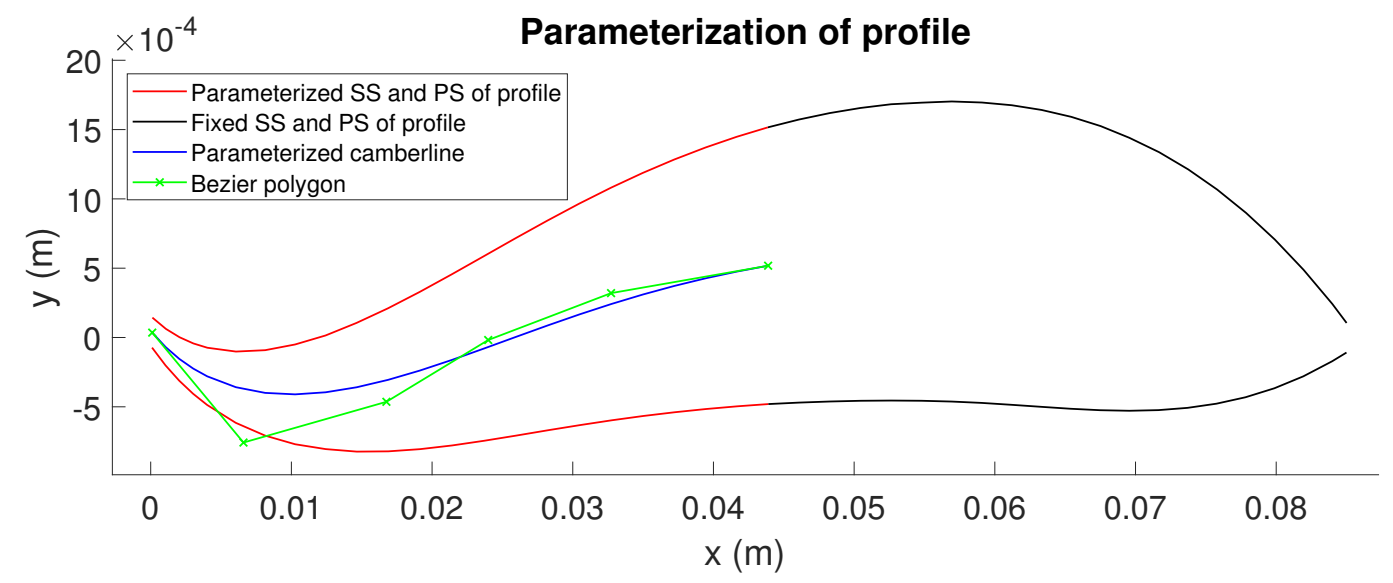

Figure 5. Parameterization of the baseline profile. Y-axis amplified for clarity. 


\subsection{Genetic Algorithm}

The optimization procedure was set up in the Matlab ${ }^{\text {TM }}$ (The MathWorks Inc., Matlab, Natick, MA, USA) environment, interfacing the multi-objective optimization algorithm with the mesher and the CFD solver. The loop was initialized with the initial population, Upper Bounds (UB) and Lower Bounds (LB) of the decision variables (decvar). The genetic algorithm sent the decvar to the script in charge of calculating the fitness functions, which in turn generated the profile, inserted it in the structured grid via a journal file, saved the case file for the CFD solver, and sent it to the UI script and maximum $p_{2}$ script, respectively. These two scripts ran a series of simulations and used reports for the quantities of interest to update the boundary conditions automatically via journal files; a more thorough description will be given soon. Once all the simulations were carried out, the value of the fitness functions were sent back to the genetic algorithm, and a new decision variable vector was generated. For this optimization problem, a genetic multi-objective algorithm was found to be a suitable option, given its ability to couple a broad exploration of variable space, thanks to its stochastic nature, to deterministic aspects, apt at focusing on the global minima of the problem. The algorithm was an NSGA-II, described in [19], employing tournament selection, scattered crossover, and Gaussian mutation, with shrink and scale factors equal to one. The scheme of the optimization framework, including fitness function blocks, is depicted in Figure 6.

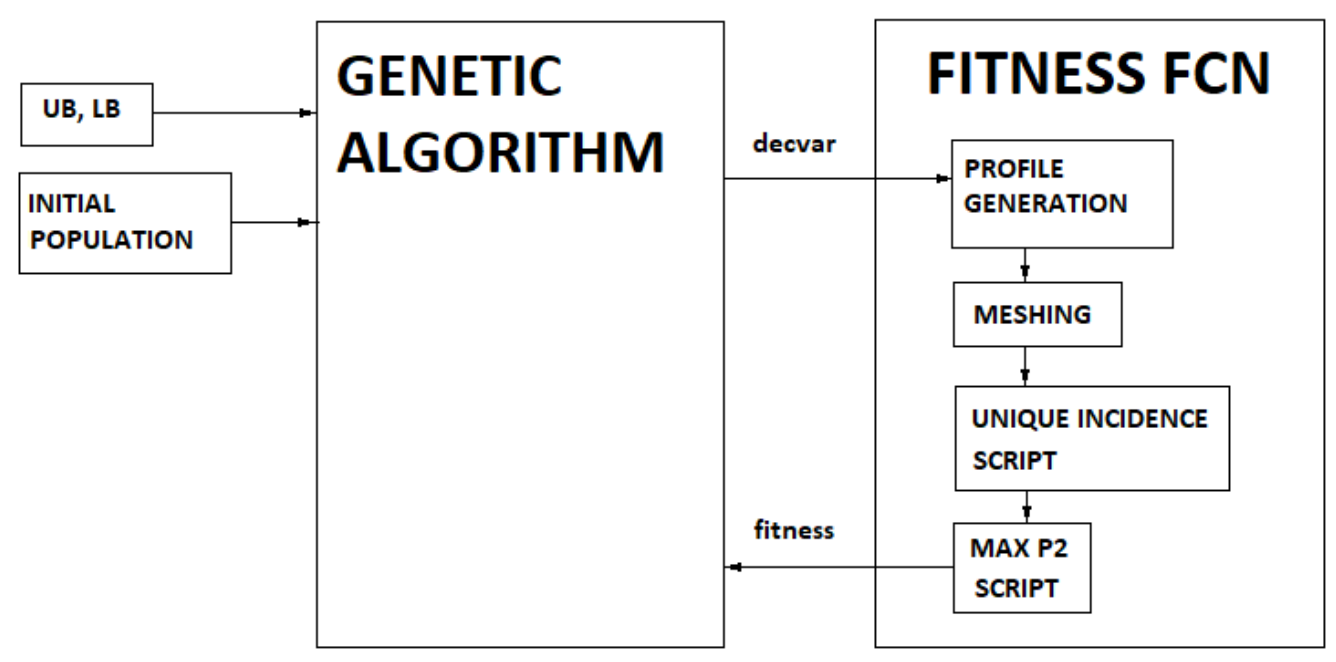

Figure 6. Scheme of the optimization framework.

\subsection{Unique Incidence Script}

In a compressor cascade with thin, low cambered profiles, under the hypothesis of supersonic inlet flow and subsonic axial velocity, the relation of unique incidence holds true, and the inlet Mach number depends on the inlet flow angle. The imposition of an arbitrary couple $\left(M_{\infty}, \beta_{\infty}\right)$ leads the cascade to operate at different inlet conditions for both variables, on a point belonging to the unique incidence curve. To obtain the desired $M_{1 \text { target }}$, an iterative procedure (inspired by [2] and described in Figure 7) was adopted, in which a low back-pressure was firstly imposed, in order to not influence the inlet flow, along with a tentative initial value for $\beta_{1}$ and the target inlet Mach number $M_{1 \text { target }}$. The $\beta_{1 \text { sim }}$ calculated by the CFD solver was set for the next simulation: it was observed that the $\beta_{1 \text { sim }}$ was always closer to the unique incidence value than the arbitrarily-chosen $\beta_{1}$. After the first two iterations, the update algorithm changed: since the inlet parameters returned by the simulations lay on the unique incidence curve, which is linear in the plane $M_{1}-\beta_{1}$ with very good approximation, the last two points were used to calculate the slope of the UI line and to set the $\beta_{1}$ for the next simulation according to a linear model. The procedure was repeated until convergence on the control parameter $\Omega$ 
was reached. On average, 3-4 simulations were requested by this algorithm for reaching convergence, against the five using simple substitution, with a tolerance toll on $\Omega$ equal to $5 \times 10^{-4}$.

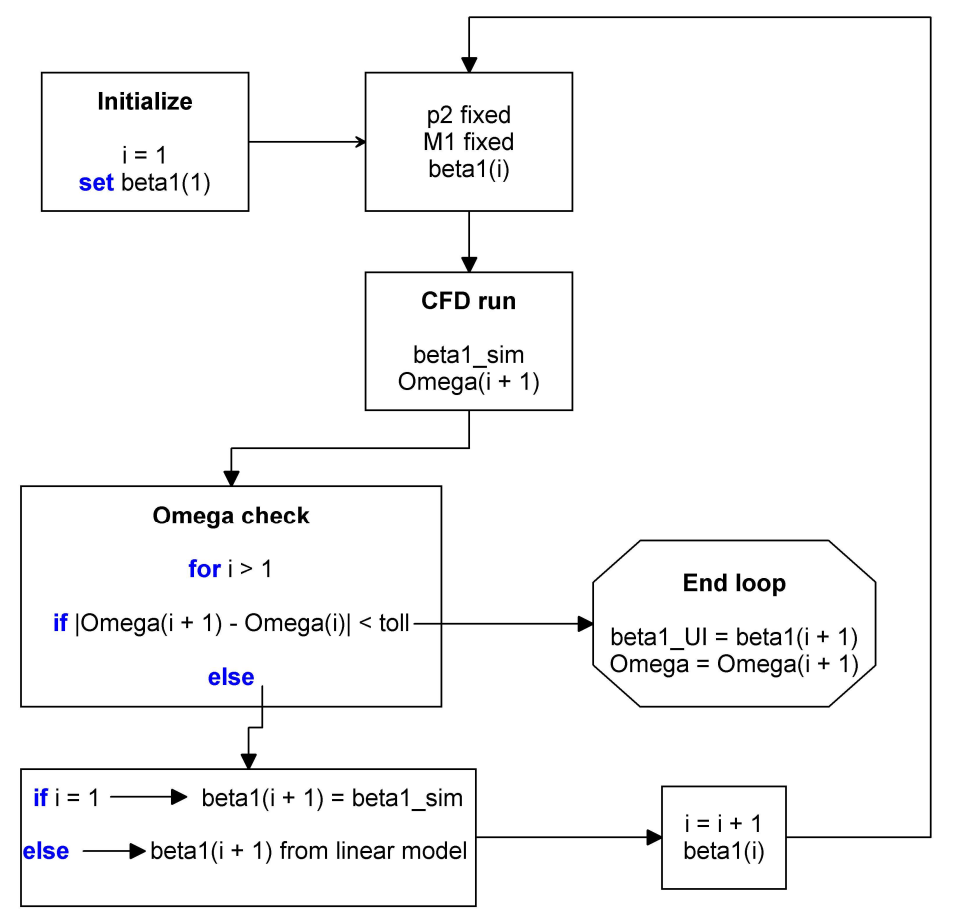

Figure 7. Loop for searching for unique incidence conditions.

\subsection{Maximum Pressure Ratio Script}

Once the unique incidence condition was found (i.e., the $\beta_{1}$ corresponding to the fixed $M_{1 \text { target }}$ ), a new procedure to find the maximum reachable pressure ratio before the spill point was launched. The concept is to bring back-pressure as close to the unstart value as possible: as soon as the spill point is reached, the influence of $p_{2}$ extends up until the inlet region (i.e., varying all relevant quantities in this section, decreasing pressure ratio and dramatically deteriorating the loss coefficient). This variation was exploited to detect the UI/unstarted status of the cascade. The algorithm is described in Figure 8: first, a back-pressure, higher than the value in the unique incidence script, was set, and then, it was verified whether inlet quantities returned by the simulation remained unchanged or not; in particular, the angle $\beta_{1}$ must stay within a narrow range centered in $\beta_{1 U I}$. If that holds true, the cascade is still in unique incidence and the back-pressure is raised by one step; otherwise, the variation of the inlet flow angle is interpreted as a spill point operating condition, and the pressure is lowered by the same step. In the next simulation, the step was halved and the procedure repeated for a fixed number of iterations $n_{\text {iter }}$. The main advantage of this algorithm is robustness: the searching procedure is based on numerical simulation and a proper choice of the number of iterations, and the initial step size is the key to explore the back-pressure range adequately. A disadvantage is that, given the number of iterations (linked to the computational time), the accuracy in calculating the objective function is inversely proportional to the explorable range amplitude. To provide the procedure with a better accuracy in the determination of the effective unstart back-pressure, a new scheme was implemented. At Mach 1.3, the scheme uses the linear trend of inlet flow angle with respect to $p_{2}$ beyond the spill point: the $p_{2}$ found at the intersection between this line and the line of UI in the $p_{2}-\beta_{1}$ space is used as a starting point for two iterations more using the bisection method, with a lower tolerance on the control parameter. At higher Mach numbers, a discontinuity in the trend of $\beta_{1}$ with respect to $p_{2}$ 
beyond the SP makes this linear method unusable. In these cases, the bisection method is carried on for three iterations more.

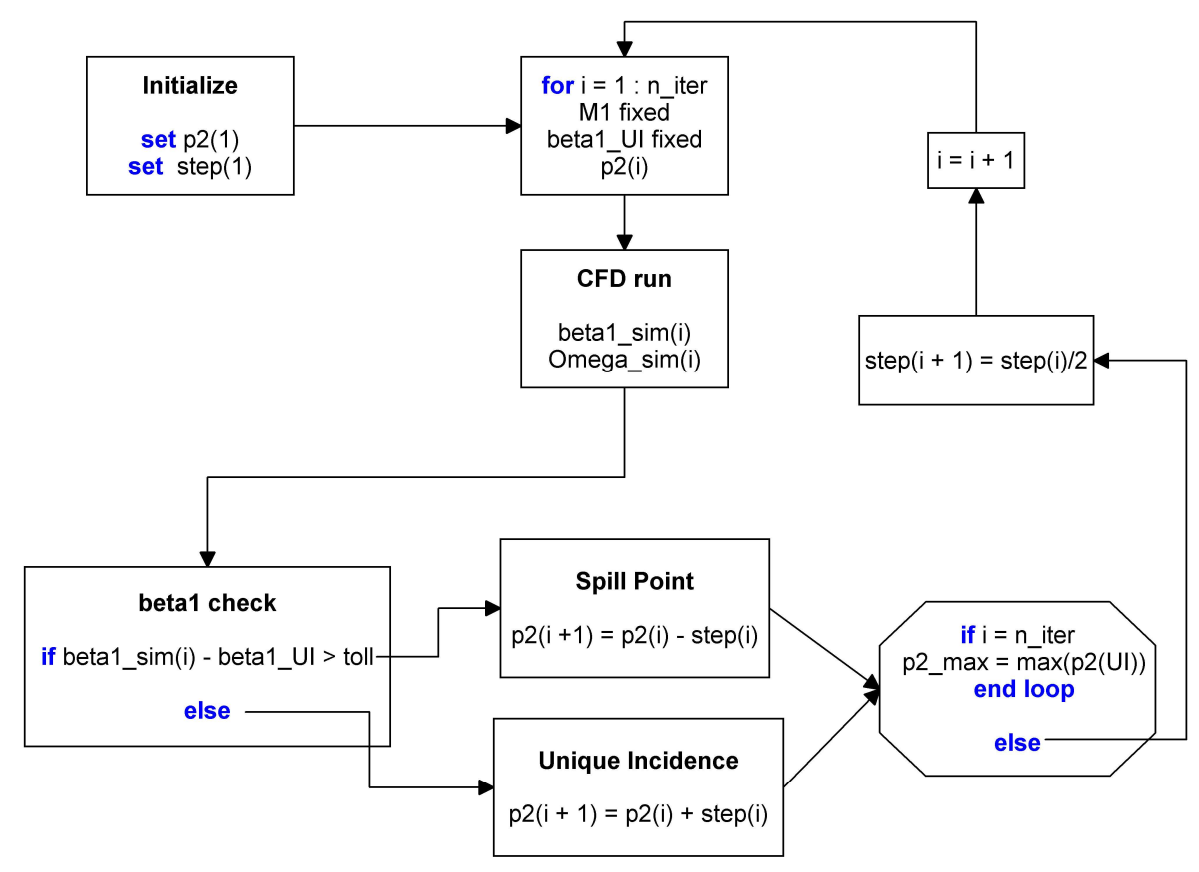

Figure 8. Maximum pressure ratio searching procedure.

\subsection{Artificial Neural Network}

To provide a further exploration of the solution space, at the end of every optimization, an ANN was used to create a meta-model upon which a novel optimization was launched. The dataset comprised all individuals simulated along with 40 individuals from a Design Of Experiments (DOE) for each optimization. The network had 10 hidden neurons, and the Bayesian-regularization training algorithm was adopted. The dataset was divided into $70 \%$ for training, $15 \%$ for validation, and $15 \%$ for testing. A new optimization was carried out using a genetic algorithm, with the same parameters described in the previous section and evaluation of the fitness functions via the above mentioned meta-model; 80 individuals were evolved for 80 generations. Profiles with the best fitness were simulated via CFD to verify predicted performance and are plotted along the Pareto fronts for each inlet Mach number in Figure 9. It can be noted that ANN was able to further enlarge the Pareto front only at Mach 1.3, probably for the insufficient size of the training dataset.

\section{Results and Discussion}

Three optimizations were conducted using three different inlet Mach numbers, namely 1.3, 1.45, and 1.58; other quantities of interest were kept unchanged in order to simulate flows with different inlet velocities, but constant inlet pressure and temperature. Each optimization used a selection of individuals from a Halton distribution as the initial population; 20 individuals for eight generations for the first $M_{1}, 10$ generations for the others, were simulated. Using an Intel Xeon CPU E5-2650 @ 2.00 GHz (Intel Corporation, Santa Clara, CA, USA) , every generation took a mean of 14 hours to be completed. The best individuals obtained for a given operative Mach number were simulated at the other two Mach numbers as well in order to test the actual need for a variable geometry to achieve better performance at different operative conditions. At the end of each optimization, a new procedure was launched, where the calculation of fitness functions was performed via ANN as stated in the 
previous section. The best individuals were simulated using CFD to ensure that meta-model prediction was accurate: the overall results are clustered in Figure 9 and in Table 7.
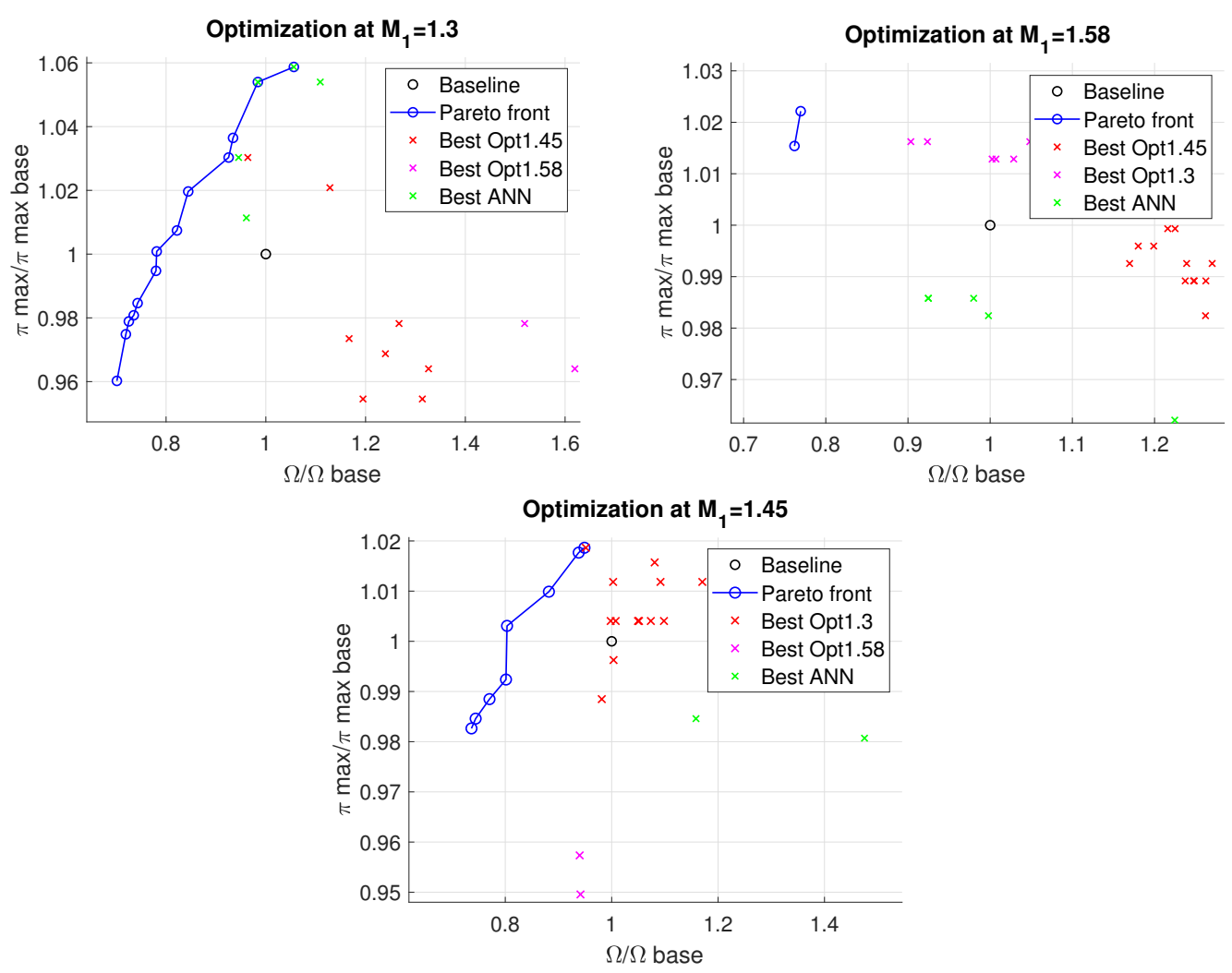

Figure 9. Pareto front of the optimizations, best individuals at other $M_{1}$ (Best Opt) and best individuals obtained via ANN (Best ANN).

Table 7. Performances of the optimized profiles. LLC, Low Loss Coefficient; OPT, Optimized profile; HPR, High Pressure Ratio; norm., normalized to baseline.

\begin{tabular}{|c|c|c|c|c|}
\hline Optimized Profiles & $\Omega$ & $\pi_{c}$ & $\Omega$ Norm. $\quad(\%)$ & $\pi_{c}$ norm. $(\%)$ \\
\hline LLC M1.3 & 0.0527 & 1.733 & 70.1 & 96.0 \\
\hline LLC M1.45 & 0.0749 & 2.158 & 73.6 & 98.3 \\
\hline OPT M1.58 & 0.1013 & 2.582 & 76.5 & 102.2 \\
\hline HPR M1.3 & 0.0793 & 1.911 & 105.6 & 105.9 \\
\hline HPR M1.45 & 0.0965 & 2.237 & 94.9 & 102.0 \\
\hline
\end{tabular}

\subsection{General Considerations}

A significant improvement in the efficiency was achieved in every optimization, with up to a $29.9 \%$ reduction of the baseline's $\Omega$, while the increment in the static pressure ratio was smaller, reaching a maximum gain of $5.9 \%$. The best individuals followed a peculiar trend, moving from Low Loss Coefficient (LLC) shapes to High Pressure Ratio (HPR) ones, as described in detail in the following. In the optimization at Mach 1.58, the Pareto front shrank to only two individuals featuring similar geometrical properties and flow fields. The efficiency rise could be explained using three main arguments: reduction of the pre-shock Mach number, increased slope of the lower branch of the bow shock, and mitigation of the strong boundary layer-shock interaction. Enhancements of the pressure ratio at lower speed regimes were due to increased positive camber and flow deflection, along with stronger bow shock intensity. Optimized shapes were investigated by means of the isentropic Mach number distribution, Mach number contours, and total pressure contours. Two optimal shapes were 
taken into account for comparison for the first two optimizations, only one for the third, given the similarities between fittest individuals on the Pareto front at Mach 1.58.

\subsection{Analysis of Profiles with the Low Loss Coefficient}

A closer look at the optimized shapes at $M_{1}=1.3$ and $M_{1}=1.45$ in Figure 10 reveals a flatter camberline in comparison to the baseline, with higher Y-coordinates for the leading edges and reduced concavity between $10 \%$ and $50 \%$ of the chord. These geometrical features become less pronounced with the increase of $M_{1}$, leading to more concave shapes similar to the baseline, but with the point of maximum curvature shifted progressively from the leading fraction towards the centerline. The optimized shape at Mach 1.58 followed this trend, keeping the efficiency high, while retaining a good pressure ratio. Looking at the Mach number contours in Figure 11, it can be noted that the baseline profile was not optimized for the operation at an inlet Mach number of 1.3. The inlet section of the flow field was characterized by a curved bow shock, slightly detached from the leading edge even in unique incidence. At all operative conditions, the local positive slope of the first $10 \%$ of the chord induced an intense flow acceleration on the suction side, with a Prandtl-Meyer expansion fan propagating up until the upper branch of the next profile's bow shock (see Figures 11-13). High efficiency profiles had a flatter camberline, reducing on the one hand the intensity of the expansion fan and increasing the obliquity of lower branch of bow shock, with lower shock losses on the other. Less sloped frontal portions of the camberline are consistent with generally lower UI angles: this aspect is particularly relevant at lower supersonic speeds, because the fraction of losses due to shocks is slight in comparison to viscous dissipation (see Figures 14 and 15), and a lower incidence reduces separation phenomena and, in the last analysis, overall losses. Reduced incidence also implies a higher mass-flow-ratio for the given inlet Mach number, but with maximum differences $<6 \%$. The flow field was divided into three zones for a more accurate analysis.

\subsubsection{From $0-20 \%$ of Chord}

Optimized shapes have a flatter leading fraction of the camberline, as can be noted in Figure 10: this aspect reduces dramatically the acceleration of supersonic flow on the suction side right behind the leading edge, related to the strong deviation imposed by the positive slope of the profile. The geometry called a "bowl shape" (i.e., the camberline had a positive slope from $0 \%-15 \%$ of chord, then decreased towards negative values) is linked to the need for a concave front portion of the chord, essential to actuate the external precompression mechanism of S-shaped profiles, reducing shock losses at high inlet Mach numbers; but at lower speeds, it becomes detrimental. The containment of this acceleration reduces the Prandtl-Meyer expansion fan, which is responsible for the increase in the pre-shock Mach number of the upper bow shock branch, as can be noted in Mach number contours in Figures 11-13. In optimized profiles, a flatter camberline yielded an attached and more sloped oblique shock at all operative conditions: in the baseline at Mach 1.3, it was curved and slightly detached even in unique incidence. At higher speeds, the increased slope led to reduced total pressure losses, as can be noted in the total pressure contours in Figures 15 and 16. 

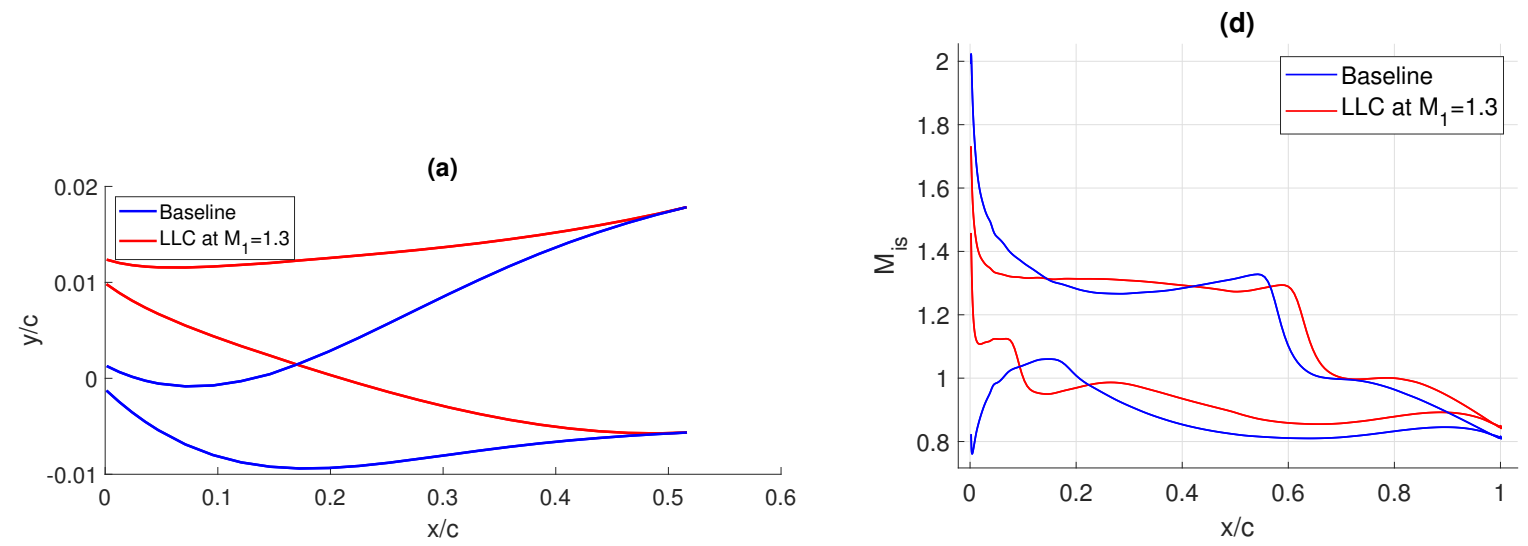

(e)
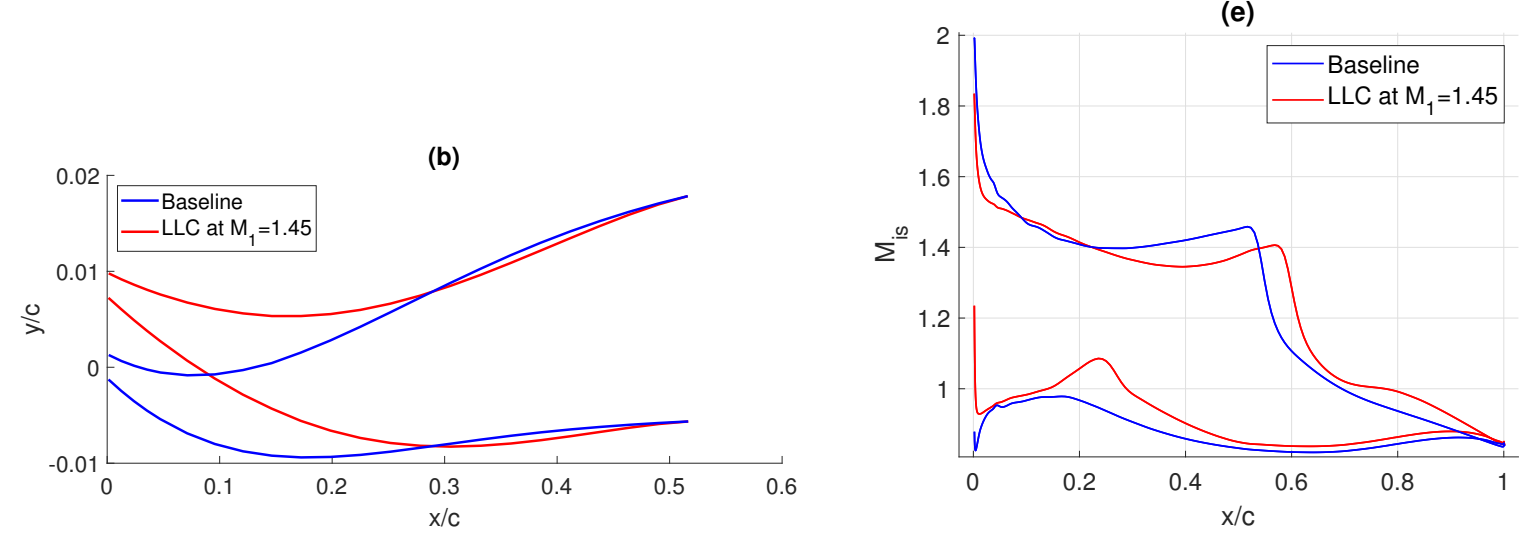

(f)

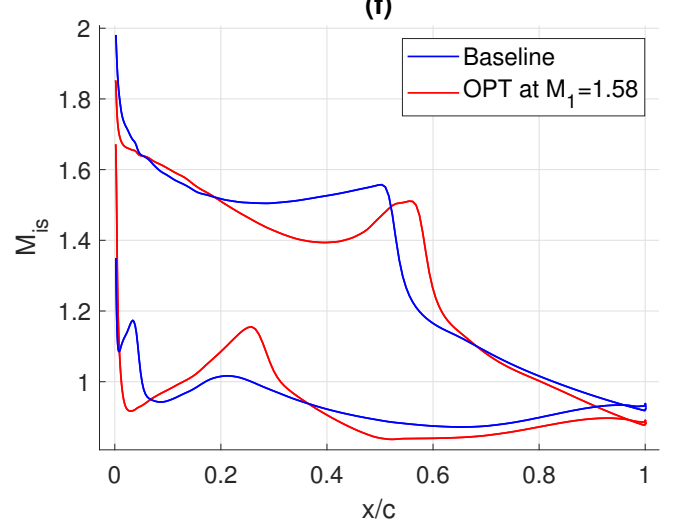

(c)

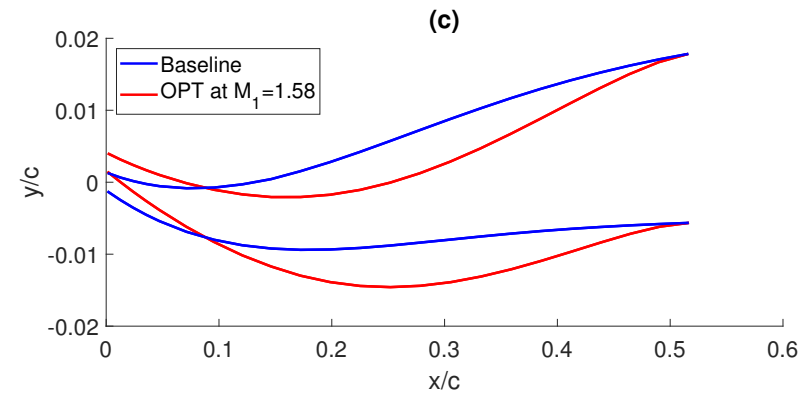

Figure 10. Front fraction shapes (a-c) and isentropic Mach number $(\mathbf{d}-\mathbf{f})$ of LLC profiles.

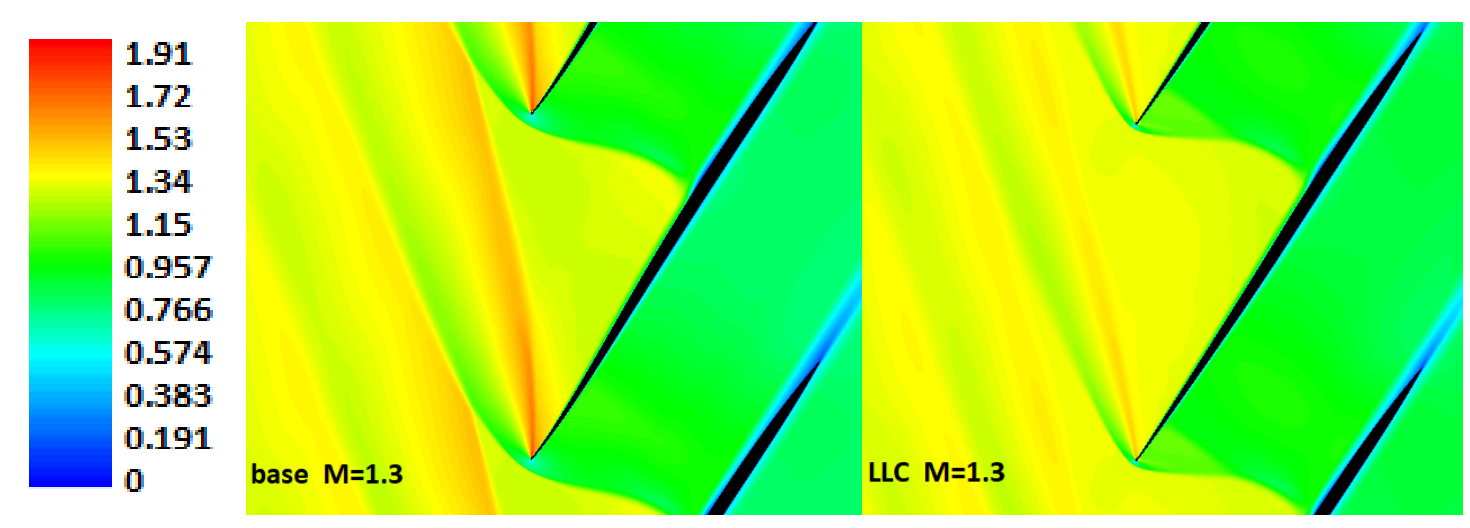

Figure 11. Mach contours of profiles with LLC at Mach 1.3. 

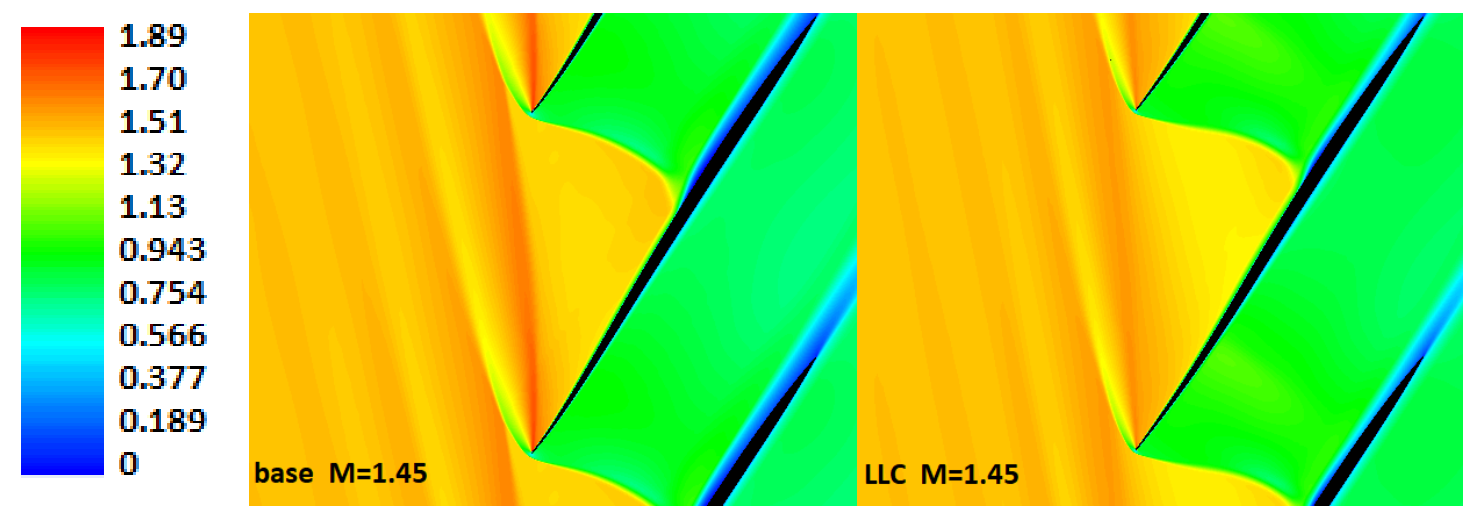

Figure 12. Mach contours of profiles with LLC at Mach 1.45.
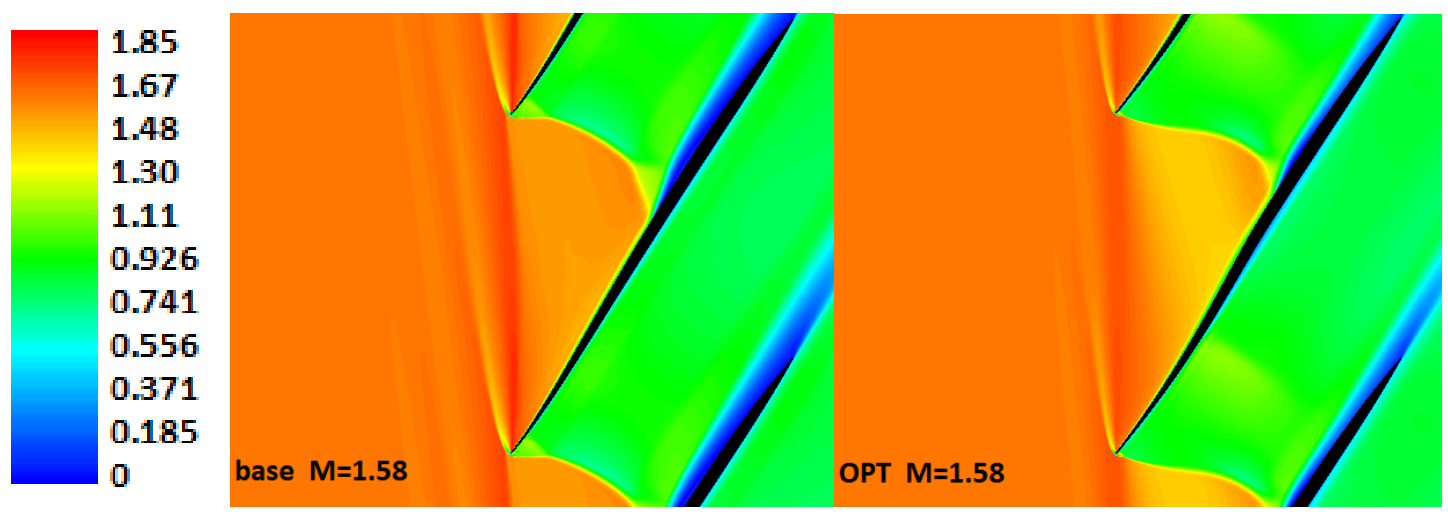

Figure 13. Mach contours of optimized profile at Mach 1.58.

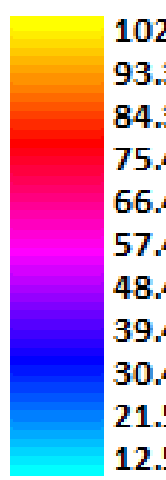

(kPa)

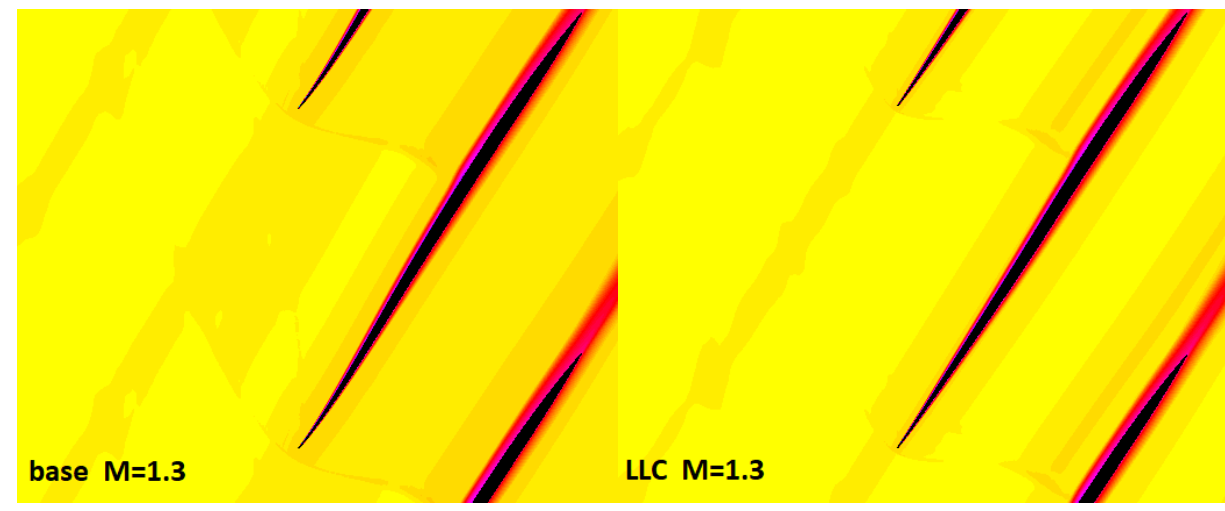

Figure 14. Total pressure contours $(\mathrm{kPa})$ of profiles with LLC at Mach 1.3.

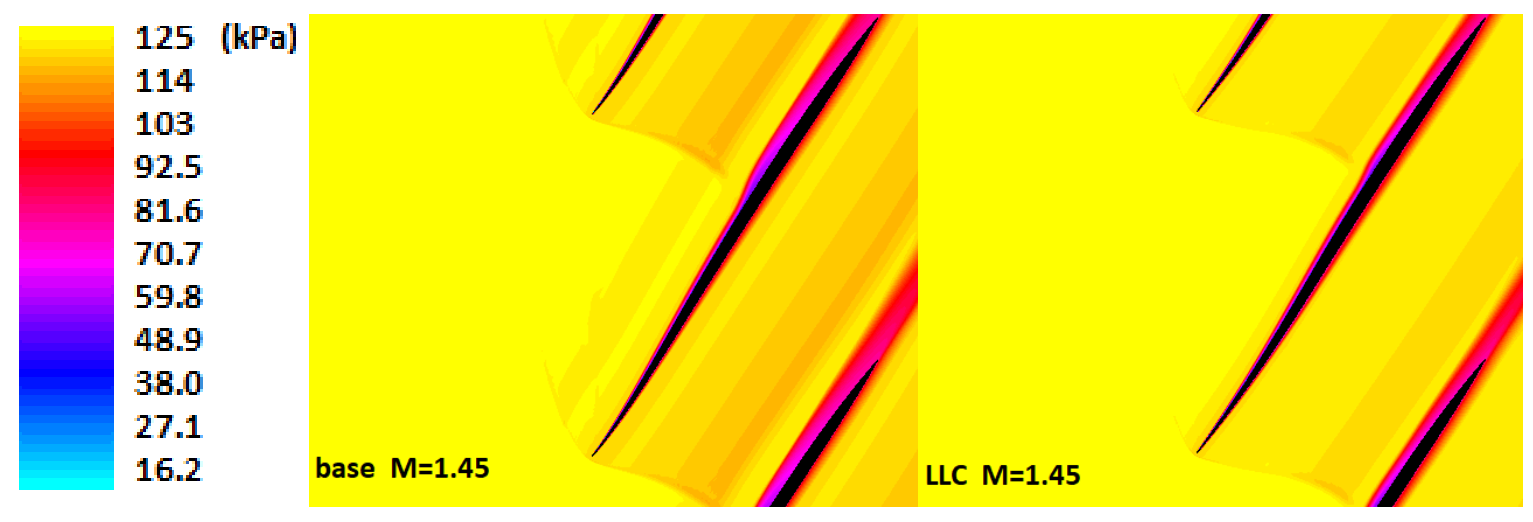

Figure 15. Total pressure contours (kPa) of profiles with LLC at Mach 1.45. 


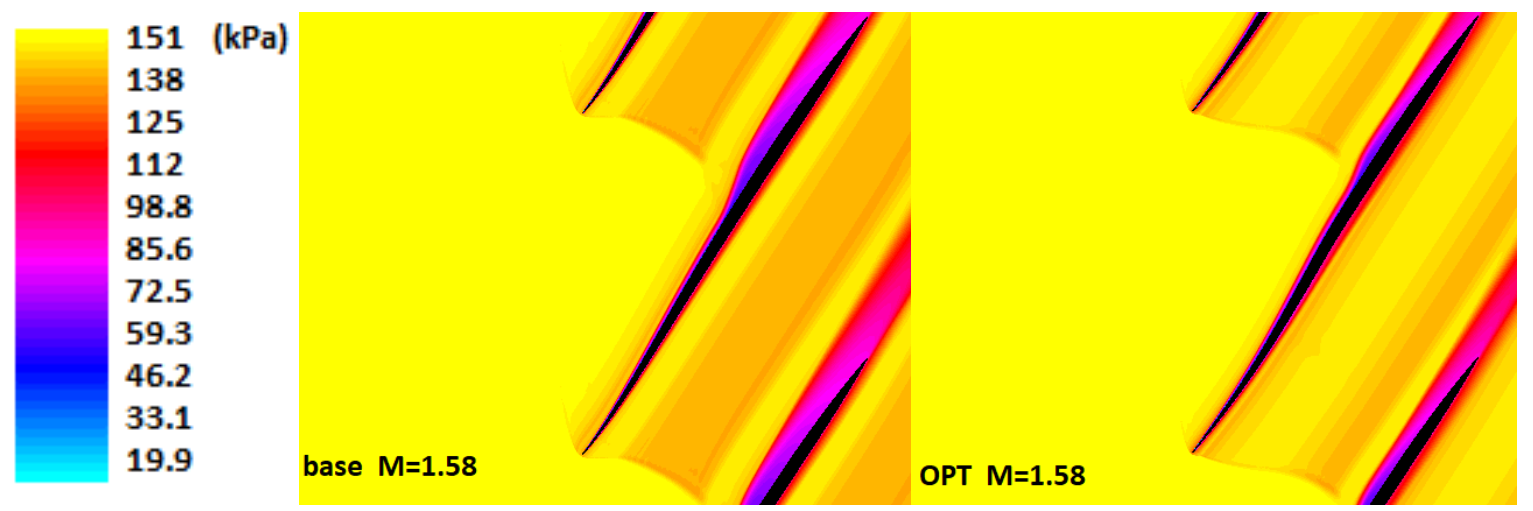

Figure 16. Total pressure contours $(\mathrm{kPa})$ of optimized profile at Mach 1.58.

\subsubsection{From $20 \%$ of Chord to Passage Shock}

In LLC shapes, this fraction of chord was concave, generating additional Mach lines and thus reducing the flow speed further, while the baseline had a flatter suction side and kept the isentropic Mach number almost constant until the intersection of the passage shock (see Figure 10). Those Mach lines covered a wide area in front of the passage shock, reducing the pre-shock Mach number up until 0.18 (see Figure 13). The impinging point between the wave and the Suction Side (SS) was located at a higher fraction of chord in optimized shapes thanks to the increased slope of the passage shock. The baseline profile presented a change in curvature, from concave to convex, at $30 \%$ of chord, responsible for the re-acceleration of the flow before passage shock incidence, that was mitigated in the LLC shapes. Total pressure losses on the suction side decreased thanks to the reduced bow shock intensity. On the pressure side, the mean isentropic Mach number was slightly higher compared to the baseline for the convex curvature, and could exceed unity, but it was turned subsonic without shocks. This aspect was due to the constancy of thickness, forcing the Pressure Side (PS) to follow modifications of SS, which had greater influence on the cascade performance.

\subsubsection{From Passage Shock to Trailing Edge}

The most evident distinctive feature in this zone was the reduced intensity of SWBLI, giving rise to less prominent separation phenomena. This aspect was related to both the weaker oblique shocks and the reduced incidence and deviation imposed on the flow by the optimized shapes, and it is of utmost importance in containing profile losses, especially at Mach 1.58, where the lambda interaction gives rise to strong separation phenomena in the baseline profile (Figures 13 and 16). Out of this, flow fields were very similar for both the baseline and optimized profile, given the unchanged shape of the rear part.

\subsection{Analysis of Profiles with a High Pressure Ratio}

Profiles optimized for higher pressure ratios in the first two optimizations had overall higher camber compared to the baseline, their leading edges being shifted below. They had a peculiar shape featuring two inflection points (see Figure 17): the first one located at $10 \%$ of the camberline, the second at half chord, similar to the baseline. The first $10 \%$ of the camberline had a negative slope and a convex curvature, and the following development of the mean line presented a strong concavity until the centerline, where the second inflection point was located. This solution may be considered a hybrid between typical supersonic geometries like the S-shapes and more conventional moderately supersonic profiles like the Multiple Circular Arc (MCA). It allowed for a considerable turning of the flow for a supersonic profile, while retaining the precompression mechanism for minimizing losses even below the baseline level. Just as already observed for LLC profiles, the optimized shapes became more similar to the baseline profile while shifting towards higher Mach numbers. Yet, it is worth noting that an individual on the front of the first optimization lied on the Pareto front of 
second optimization as well. This peculiar shape disappeared in the Pareto front at Mach 1.58, being dominated by more conventional designs for both objectives, probably because of an insufficient precompression. The higher difference in isentropic Mach number between the Suction Side (SS) and Pressure Side (PS) in HPR shapes gave evidence of the higher loading, while peak values remained substantially lower compared to the baseline (see Figure 17). Profiles with two inflection points showed a higher unique incidence angle and, thus, a reduced mass flow ratio, with a maximum variation of $10 \%$ compared to the baseline.
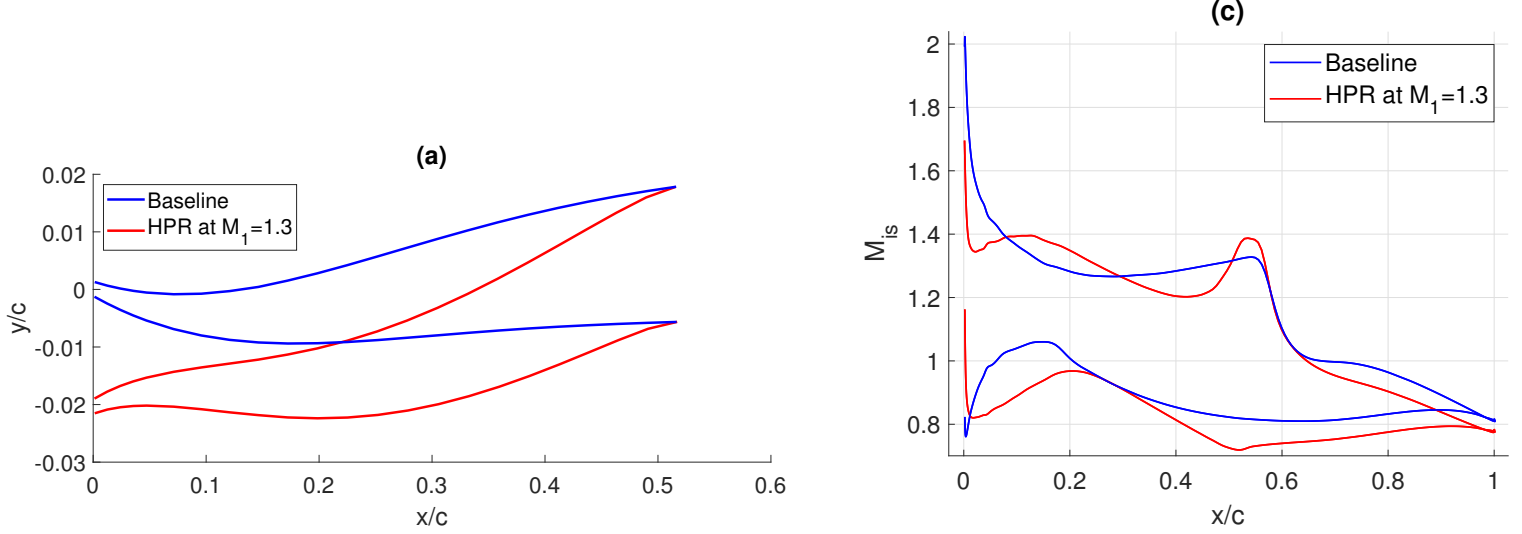

(d)
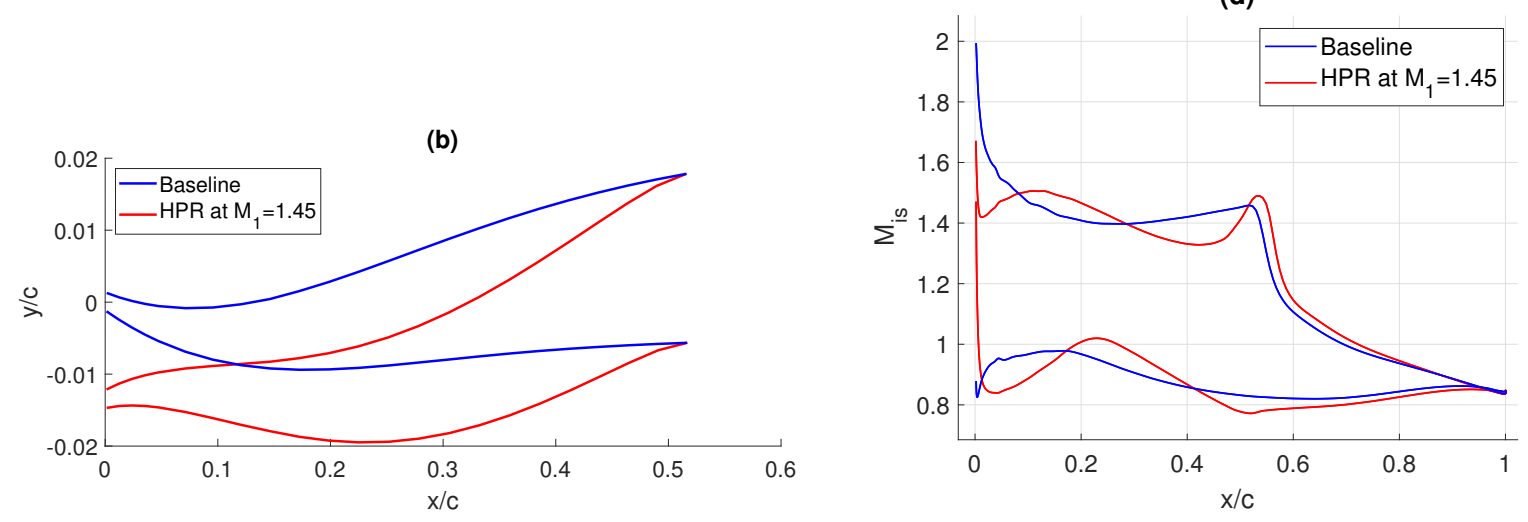

Figure 17. Front fraction shapes $(\mathbf{a}, \mathbf{b})$ and isentropic Mach number $(\mathbf{c}, \mathbf{d})$ of HPR profiles at Mach 1.3 and 1.45 .

\subsubsection{From $0-20 \%$ of Chord}

Looking at Figures 18 and 19, it can be noticed that the bow shock had a shape comparable to the baseline, the main difference downstream being the disappearance of the strong expansion fan due to the negative slope of the front fraction of the chord, which followed the streamlines and caused reduced deviation of flow on the suction side. This negative slope allowed the shock to remain attached with increased incidence and deviation, incrementing the profile loading without affecting the losses much. The passage oblique shock was more normal in profile with HPR, with higher losses, but also a reduced sensitivity on back-pressure variation [2], allowing for a greater margin before spill point insurgency. Two expansion fans radiated from both the SS and the PS near the leading edge, but with peaks below the baseline values. The latter could reduce the sensitivity of the passage shock to back-pressure rising, as suggested in [2]. The upper band of the passage shock was subjected to lower pre-shock Mach numbers, thanks to the pre-compression mechanism, reducing shock losses. 

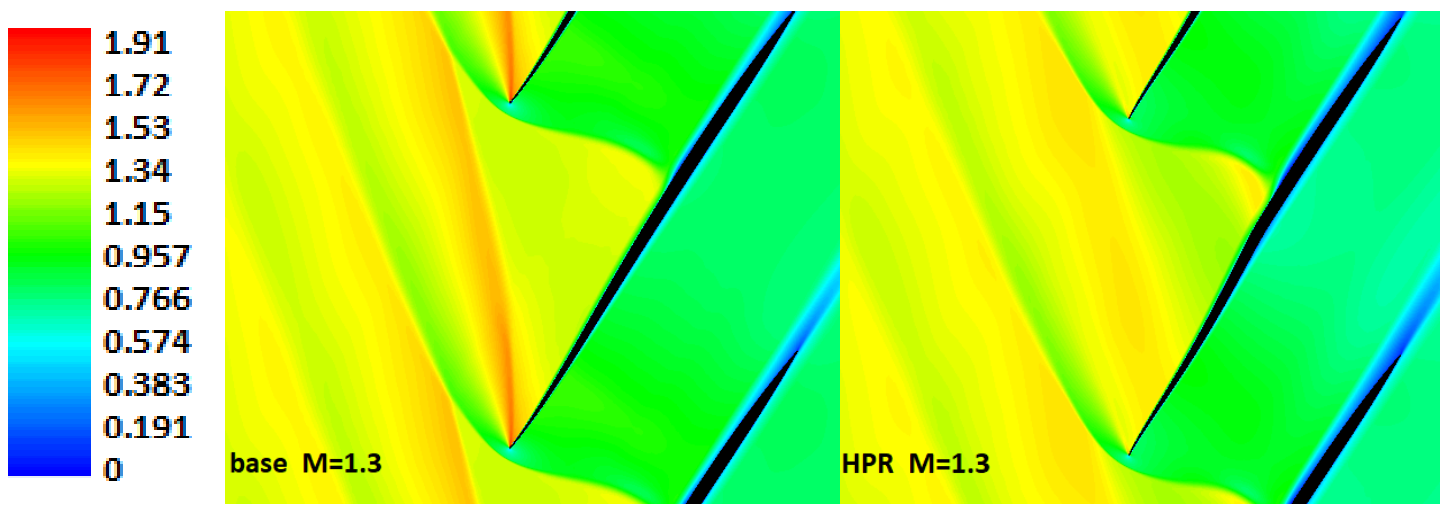

Figure 18. Mach contours of the profiles with HPR at Mach 1.3.
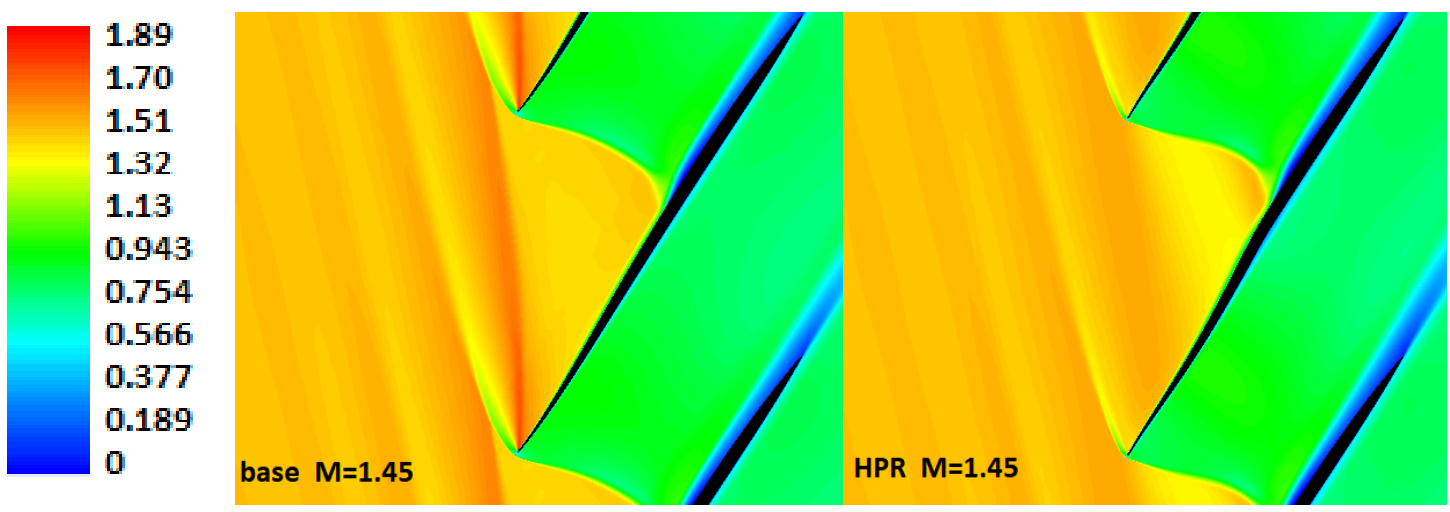

Figure 19. Mach contours of profiles with HPR at Mach 1.45.

\subsubsection{From $20 \%$ of Chord to Passage Shock}

Concavity subsisted on the suction side for a longer fraction of chord compared to the baseline, bringing about a higher deceleration and the propagation of a wide left-running characteristics fan, propagating in front of the next profile's passage shock. The very large radius of curvature prevented the fan from coalescing into a single wave, reducing the production of entropy. The following rapid change of curvature accelerated the flow again, leading to a higher pre-shock Mach number compared to the baseline and to a more intense shock, but only in the terminal band, as one can see in Figures 18-21. The adverse pressure gradient on the suction side thickened the boundary layer, but without detachment. On the pressure side, the divergence of area brought about a further deceleration of the subsonic flow maintaining lower speeds than the baseline.

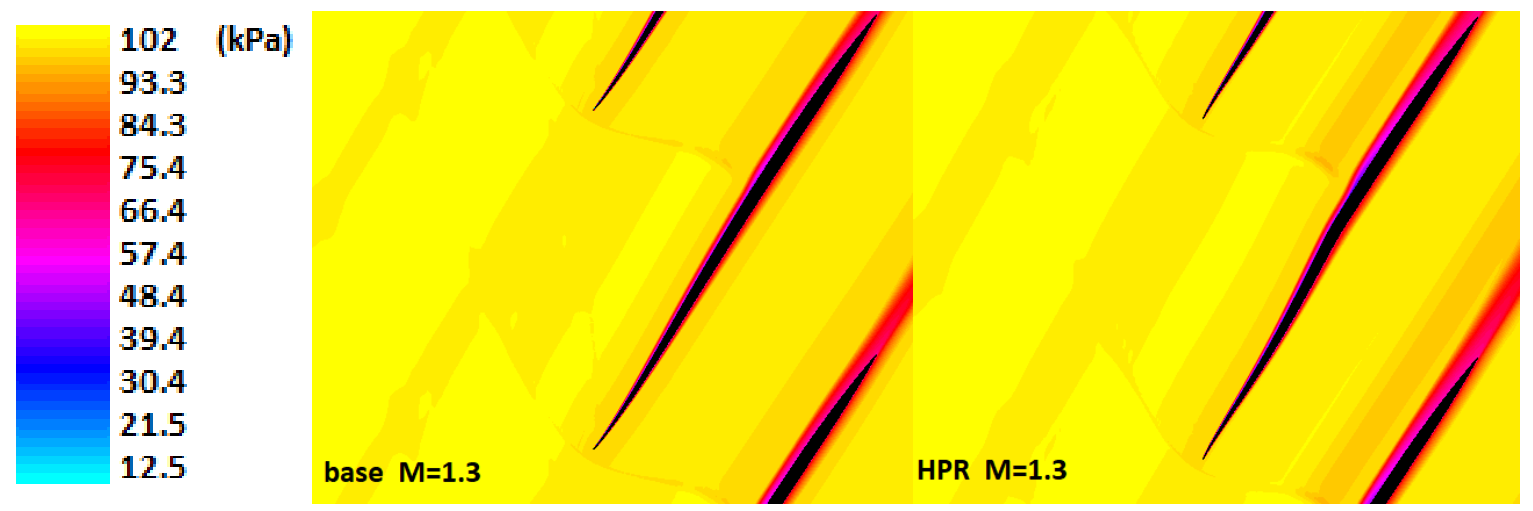

Figure 20. Total pressure contours $(\mathrm{kPa})$ of profiles with HPR at Mach 1.3. 


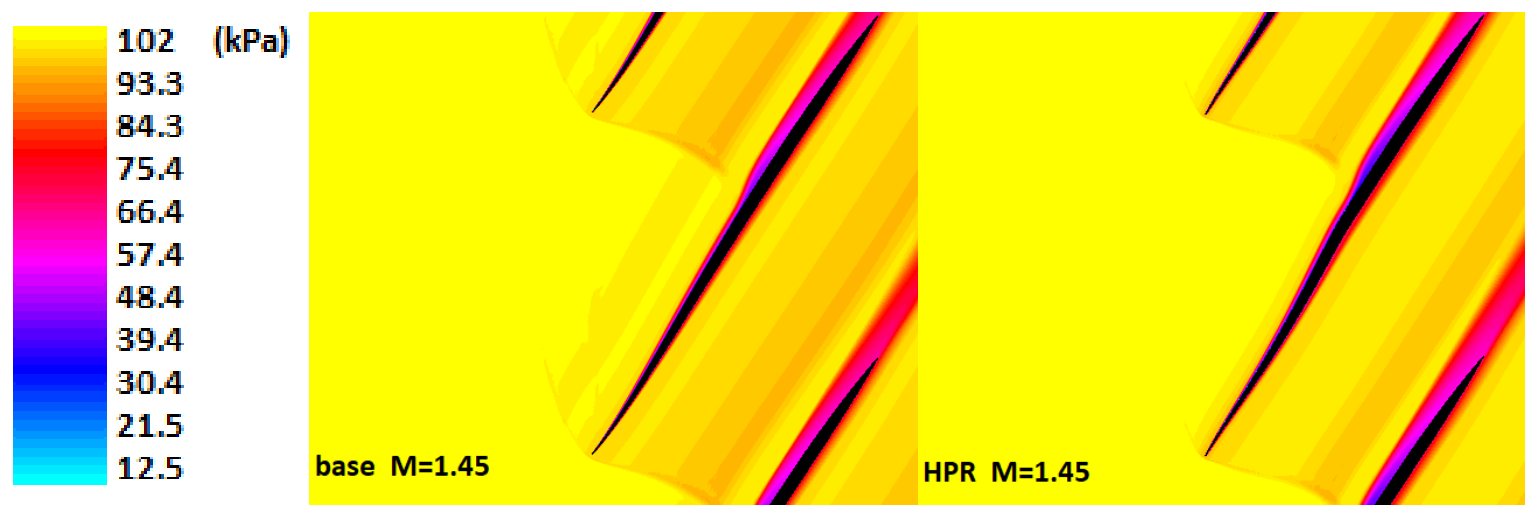

Figure 21. Total pressure contours (kPa) of profiles with HPR at Mach 1.45.

\subsubsection{From Passage Shock to Trailing Edge}

The interaction between shock and boundary layer was slightly more intense, giving rise to a stronger detachment on optimized shapes. Mean velocities until the trailing edge were lower because of the higher back-pressure.

\section{Conclusions}

The multi-objective, multi-point optimization showed that there is a substantial margin of improvement for efficiency (up to 29\%) and a lower one for pressure ratio (up to $5.9 \%$ ) at all operative Mach numbers, including the closest to nominal operative condition. The improvement in the loss coefficient at Mach 1.45 of $26.4 \%$ exceeded $25 \%$ (obtained by [2]) for the DLR-PAV-1.5 cascade at the same inlet Mach number, despite the higher computational cost of the algorithm adopted in [2]. A greater enhancement (25.5\%) was recorded at Mach 1.58 compared to the $20 \%$ achieved by [3] at the same Mach number with the ARL-SL19 cascade. The profile was parametrized only for the first half of the camberline without limiting the performance enhancement, confirming its primary relevance in supersonic compressor profiles. It was observed that optimized shapes for a specific Mach number differed significantly from optimal at different conditions, proving the high sensitivity to the off-design of supersonic cascades. Two families of optimized geometries were discovered: the first one achieved greater efficiency, reducing the bowl shape near the leading edge and the related expansion fan, increasing passage shock slope and reducing interaction between the boundary layer and shock wave. The concave curvature on the suction side became more prominent in designs optimized for higher speeds, where the precompression mechanism was more relevant, but it was shifted towards aft compared to the baseline. All the LLC profiles featured lower incidence compared to the baseline, and thus lower separation and losses. At lower Mach numbers, a flatter camberline seemed to be responsible for the reduced inlet flow angle, while at higher speeds, the reduction was achieved by more complex shapes with higher concavity near the centerline: further investigations are requested to better understand the mechanism involved. The second geometry investigated reached better pressure ratios and increased deviation, while keeping the efficiency at the baseline level. It presented a more cambered shape, providing higher loading, and retained a good loss coefficient by reducing the acceleration of flow before the passage shock with a negative slope near the leading edge followed by a concave portion of the suction side. This geometry featured a more "normal" passage shock structure, less susceptible to back-pressure rising, but its performance worsened at Mach 1.58, where it was dominated in both the objectives by more conventional designs. This work proved the relevant enhancement in cascade performance achievable with a relatively low computational effort and a stochastic algorithm. The results obtained lead the way to more complex analyses towards expanding Pareto fronts, searching for actual global minima and, potentially, to the evaluation of variable-geometry cascade advantages and feasibility. 
Author Contributions: Conceptualization: E.B.; methodology: M.C., A.M., and E.B.; validation: M.C.; formal analysis: M.C.; writing, original draft preparation: M.C. and A.M.; writing, review and editing: A.M. and E.B.; supervision: E.B.

Funding: This research received no external funding.

Conflicts of Interest: The authors declare no conflict of interest.

\section{Abbreviations}

\begin{tabular}{|c|c|}
\hline 1 & Inlet section of the cascade \\
\hline 2 & Exit section of the cascade \\
\hline$M$ & Mach number \\
\hline$\beta$ & Flow angle relative to the $\mathrm{X}$-axis \\
\hline$p$ & Static pressure \\
\hline$p_{0}$ & Total pressure \\
\hline$T$ & Static temperature \\
\hline$T_{0}$ & Total temperature \\
\hline$\Omega$ & Total pressure loss coefficient \\
\hline$\pi_{c}$ & Static pressure ratio \\
\hline$M_{i s}$ & Isentropic Mach number \\
\hline$c$ & Chord length \\
\hline$c_{a x}$ & Axial chord length \\
\hline ANN & Artificial Neural Network \\
\hline AVDR & Axial Velocity Density Ratio \\
\hline decvar & Decision Variables \\
\hline DLR & Deutsche Zentrum für Luft und Raumfahrt \\
\hline HPR & High Pressure Ratio \\
\hline LB & Lower Bounds \\
\hline LLC & Low Loss Coefficient \\
\hline MCA & Multiple Circular Arc \\
\hline NASA & National Air and Space Administration \\
\hline NSGA & Non-dominated Sorting Genetic Algorithm \\
\hline OPT & Optimized profile \\
\hline PS & Pressure Side \\
\hline RANS & Reynolds Averaged Navier-Stokes \\
\hline REAL & Realizable \\
\hline RNG & Re-Normalized Group \\
\hline SA & Spalart-Allmaras \\
\hline $\mathrm{SP}$ & Spill Point \\
\hline SS & Suction Side \\
\hline SST & Shear Stress Transport \\
\hline STD & Standard \\
\hline SWBLI & Shock Wave-Boundary Layer Interaction \\
\hline UI & Unique Incidence \\
\hline UB & Upper Bounds \\
\hline
\end{tabular}

\section{References}

1. Chen, J.-P.; Hathaway, M.D.; Herrick, G.P. Prestall Behavior of a Transonic Axial Compressor Stage via Time-Accurate Numerical Simulation. J. Turbomach. 2008, 130, 041014. [CrossRef]

2. Venturelli, G.; Benini, E. Kriging-assisted Design Optimization of S-shape Supersonic Compressor Cascades. Aerosp. Sci. Technol. 2016, 58, 275-297. [CrossRef]

3. Liu, B.; Shi, H.; Yu, X. A New Method for Rapid Shock Loss Evaluation and Reduction for the Optimization Design of a Supersonic Compressor Cascade. Proc. Inst. Mech. Eng. Part G J. Aerosp. Eng. 2017, 232, 2458-2476. [CrossRef] 
4. Sun, X.; Yang, S.; Zhao, Q. Shock loss model and blade profile optimization design of a supersonic cascade. Proc. Inst. Mech. Eng. Part G J. Aerosp. Eng. 2015, 229, 1325-1329. [CrossRef]

5. Aissa, M.H.; Verstraete, T.; Vuik, C. Aerodynamic optimization of supersonic compressor cascade using differential evolution on GPU. AIP Conf. Proc. 2016, 1738, 480077.

6. Mohsen, M.; Owis, F.M.; Hashim, A.A. The Impact of Tandem Rotor Blades on the Performance of Transonic Axial Compressors. Aerosp. Sci. Technol. 2017, 67, 237-248. [CrossRef]

7. Yue, S.; Wang, Y.; Wang, H. Design and Optimization of Tandem Arranged Cascade in a Transonic Compressor. J. Therm. Sci. 2018, 27, 349-358. [CrossRef]

8. Tweedt, D.L.; Schreiber, H.A.; Starken, H. Experimental Investigation of the Performance of a Supersonic Compressor Cascade. ASME J. Turbomach. 1988, 110, 456-466. [CrossRef]

9. Levine, P. The Two Dimensional Inflow Conditions for a Supersonic Compressor with Curved Blades. ASME J. Appl. Mech. 1957, 24, 165-169.

10. Cumpsty, N.A. Compressor Aerodynamics; Longman Group: London, UK, 1984.

11. Lichtfuss, H.J.; Starken, H. Supersonic Cascade Flow. Prog. Aerosp. 1974, 15, 37-149. [CrossRef]

12. ANSYS Fluent User Guide; Ansys Inc.: Canonsburg, PA, USA, 2006.

13. Menter, F. Two-Equation Eddy-Viscosity Turbulence Models for Engineering Applications. AIAA J. 1994, 32, 1598-1605. [CrossRef]

14. Launder, B.E.; Spalding, D.B. The Numerical Computation of Turbulent Flows. Comput. Methods Appl. Mech. Eng. 1974, 3, 269-289. [CrossRef]

15. Shih, T.-H.; Liou, W.W.; Shabbir, A.; Yang, Z.; Zhu, J. A New $\kappa-\epsilon$ Eddy Viscosity Model for High Reynolds Number Turbulent Flows. Comput. Fluids 1995, 24, 227-238. [CrossRef]

16. Yakhot, V.; Orszag, S.A. Renormalization Group Analysis of Turbulence. I. Basic Theory. J. Sci. Comput. 1986, 1, 3-51. [CrossRef]

17. Spalart, P.; Allmaras, S. A One-Equation Turbulence Model for Aerodynamic Flows. In Proceedings of the 30th Aerospace Sciences Meeting and Exhibit, Reno, NV, USA, 6-9 January 1992; doi:10.2514/6.1992-439. [CrossRef]

18. Piovesan, T.; Magrini, A.; Benini, E. Accurate 2-D Modeling of Transonic Compressor Cascade Aerodynamics. Aerospace 2019, 6, 57. [CrossRef]

19. Konak, A.; Coit, D.W.; Smith, A.E. Multi-objective optimization using genetic algorithms: A tutorial. Reliab. Eng. Syst. Saf. 2006, 91, 992-1007. [CrossRef]

(C) 2019 by the authors. Licensee MDPI, Basel, Switzerland. This article is an open access article distributed under the terms and conditions of the Creative Commons Attribution (CC BY) license (http:/ / creativecommons.org/licenses/by/4.0/). 